\title{
POST-ACTIVATION PERFORMANCE ENHANCEMENT STRATEGIES IN SPORT: A BRIEF REVIEW FOR PRACTITIONERS
}

review paper

( ) University School of Physical Education in Wroclaw

DOI: https://doi.org/10.5114/hm.2021.103280

\section{DANIEL BOULLOSA}

${ }^{1}$ Integrated Institute of Health, Federal University of Mato Grosso do Sul, Campo Grande, Brazil

${ }^{2}$ Sport and Exercise Science, James Cook University, Townsville, Australia

\begin{abstract}
In this review, we will present and critically discuss how different conditioning exercises can be implemented in training, testing, and competition for the enhancement of performances in different sports, via post-activation performance enhancement and other delayed potentiation responses. The potentiation approaches described here include warming up, testing and monitoring, re-warm-up and priming strategies, and complex training. The post-activation performance enhancement responses can be best described following the new taxonomy, which allows the identification of the best strategies in every specific sport setting. This requires identifying the post-activation performance enhancement factors, which are the conditioning activity, the verification test, the population of athletes; and potential moderators (i.e. exercise type and loading, timing; recovery interval, target exercise, performance parameter; training background, age, and sex). The inherent limitations to these approaches, including the gaps in literature requiring further studies, may be overcome in practice by using individualized approaches.
\end{abstract}

Key words: post-activation potentiation, post-activation performance enhancement, sports physiology, warm-up, priming exercises

\section{Introduction}

The most widely cited definition of post-activation potentiation (PAP) is that proposed by Tillin and Bishop [1], in which PAP is defined as 'the phenomena by which muscular performance characteristics are acutely enhanced as a result of their contractile history'. This definition works well in any context. However, this is not an orthodox definition as PAP would necessarily be related to a twitch verification test, a point that the authors subsequently clarify in the same classic article [1]. In fact, most studies in sport have not been conducted with twitch verification tests, but with muscle power tests (e.g. vertical jumps), promoting some misconception around potentiation responses [2]. For this reason, Cuenca-Fernández et al. [3] have introduced the term 'post-activation performance enhancement' (PAPE) [3-5] to clearly differentiate between PAP and PAPE. Both PAP and PAPE are induced by a voluntary conditioning activity (CA). However, PAPE refers only to performance enhancements in voluntary exercises. Meanwhile, PAP would refer to force increases during twitch verification tests. In this regard, it is common to simultaneously identify PAP and PAPE after the same CAs [2, 6]. Therefore, as we will present only studies with athletes in sport settings, PAPE is the correct term (Figure 1). To improve the accuracy of describing the phenomena and the applicability of potentiation protocols, we will also introduce a novel taxonomy to better identify the CA, the target exercise (i.e. verification test), and the specific population [7].

PAPE is considered one of the main objectives of warm-up protocols [8], apart from the physiological benefits derived from the rise of body temperature, including enhanced neuromuscular and cardiometabolic responses $[8,9]$. The main mechanism related to potentiation has been suggested to be the phosphorylation of the regulatory light chain of myosin, a muscle memory mechanism which increases the sensitivity

Correspondence address: Daniel Boullosa, Instituto Integrado de Saúde, Universidade Federal de Mato Grosso do Sul, Cidade Universitária s/n, 79070-900, Campo Grande, Brazil, e-mail: daniel.boullosa@gmail.com

Received: October 18, 2020

Accepted for publication: December 9, 2020

Citation: Boullosa D. Post-activation performance enhancement strategies in sport: a brief review for practitioners. Hum Mov. 2021;22(3):101-109; doi: https://doi.org/10.5114/hm.2021.103280. 
A) PAP

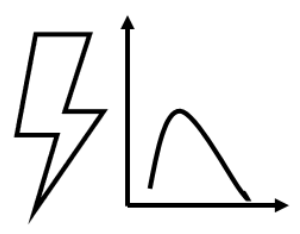

B) PAPE

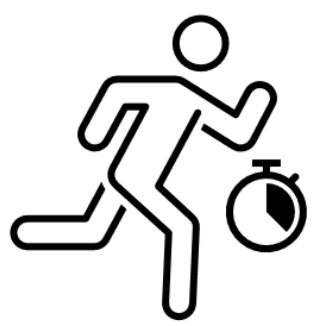

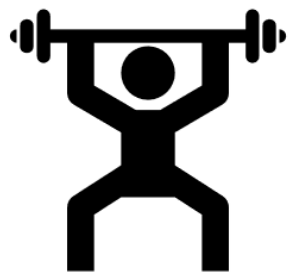

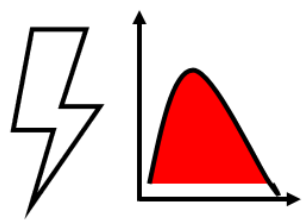

Figure 1. Schematic differences between post-activation potentiation (PAP) and post-activation performance enhancement (PAPE)
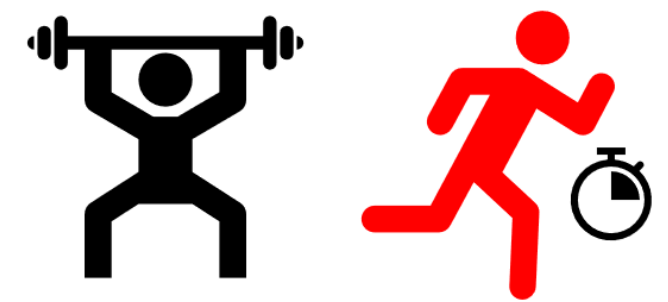

to $\mathrm{Ca}^{2+}$, thus favouring transient increments in peak force and rate of force development [10]. However, several other mechanisms have been proposed [1, 4, 6, 11]. As the scope of this review is focused on the practical aspects of PAPE, we will present and discuss the PAPE factors and their main moderators, the applications of PAPE protocols, and the usefulness of the new taxonomy to better identify efficient PAPE protocols to be applied in the sporting field for different purposes.

\section{PAPE factors and their moderators}

During the last decade, several reviews [1, 12, 13] and meta-analyses [14, 15] have provided a good approximation of the potentiation effects of different CAs and of what parameters or moderators can be manipulated for achieving the desired acute potentiation responses in every scenario. The overall conclusions from the 2 existing meta-analyses [14, 15] suggest that various sets (i.e. $2-3$ sets) of a few repetitions (i.e. $\leq 6$ repetitions) of high-intensity (i.e. $>70 \%$ of one-repetition maximum [1RM]) exercises are effective for increasing (after $>5$ minutes of recovery) performance of power exercises, such as jumps, sprints, and throws, in well-trained strength or power athletes. However, these conclusions should be taken with caution because of heterogenous study designs and sample characteristics that may limit the applicability of the findings in each specific sport setting. For instance, while the 2 meta-analyses $[14,15]$ suggest a moderate time recovery (i.e. 5-10 minutes) for greater potentiation responses, earlier (i.e. $<5$ minutes) potentiation responses have also been observed in some studies with both resistance [16, 17] and ballistic [13] exercises. More importantly, several studies have suggested the necessity of individualized approaches as the generalization of findings is often not valid for individual responses in the same studies [6, 16-18]. In this regard, if we consider our recent taxonomy proposal [7], which highlights the necessity of better identifying the main factors of PAPE as CA, the verification test, and population, it may be suggested that other potential moderators (e.g. timing) of these factors could have been ignored. In this respect, and on the basis of the aforementioned and other recent reviews on this topic [12, 19-21], we suggest the consideration of other potential moderators of PAPE factors (Figure 2).

While both the exercise type and loading parameters are the most studied moderators of CAs, there are still gaps in literature, especially considering new strategies with different exercises and loading. This applies from the most widely used such as the squat [14, 15] and ballistic [13] exercises. For instance, new approaches have been demonstrated to be effective CAs for PAPE, including different eccentric overloads [12, 22-24], elastic bands [25, 26], and blood flow restriction [27], which further expands the possibilities from classic approaches [28]. Meanwhile, the timing for implementation of the CAs (i.e. how the conditioning stimuli are distributed over time) is another moderator that has received less attention and that may include both intra-session effort distribution as set configuration [17, 29] and delayed potentiation effects as is commonly observed in in team sports with re-warm-up [20, 30] and priming strategies [21, 31]. Interestingly, although there is a body of evidence suggesting an enhancement of power performances for up to 48 hours after a CA [21], it is not clear if this delayed potentia- 


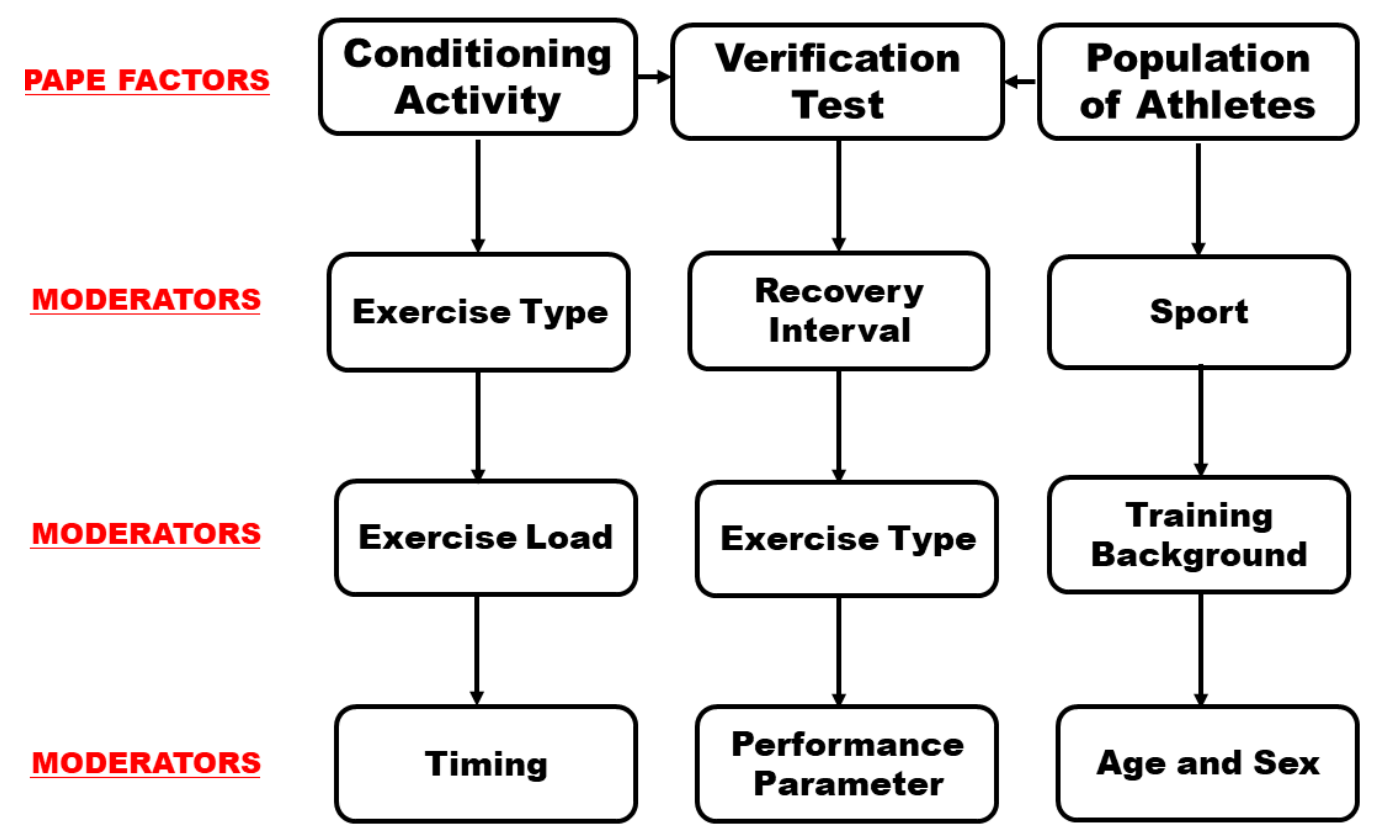

The PAPE factors are those to be included in the descriptors of the new taxonomy. The potential moderators are those characteristics associated to each factor (i.e. conditioning activity, verification test, and population) that should be considered to better individualize the implementation of a PAPE protocol.

Figure 2. Post-activation performance enhancement (PAPE) factors and their moderators

tion has some physiological association with classic PAP and PAPE mechanisms. Further studies should clarify these issues while looking for the more efficient approaches in each sport setting, especially with respect to the application of potentiation approaches requiring the use of less implements.

Regarding the moderators associated to the verification test, apart from the previously commented conflicting results regarding the best time recovery interval required for optimal PAPE, it is important to note the type of target exercise to be potentiated (i.e. verification test) and its associated performance parameters. For instance, from a practical point of view, it is recommended to identify specific PAPE responses following a particular CA, independently of PAP observed after the same CA [2]. Moreover, the enhancement of performance in cyclic vs. acyclic exercises [32], or power [14] vs. endurance [19] exercises, is another issue to be further explored as the best performance parameters are dependent upon the type of verification test. It is also dependent on the performance parameters selected, including peak vs. mean values [16, 33] comparisons (e.g. peak power vs. mean power during a countermovement jump; mean vs. peak velocity during a repetitive sprint test).

Population characteristics is likely the PAPE factor which requires the most attention. While the years of training, or strength levels, have been systematically recognized as important moderators [14, 15], it may be suggested that some of the generalizations from the literature may be biased by the potential influence of other, less studied moderators, as age and sex. Regarding sex, the findings in literature are conflicting $[13,14$, $32,34]$. As of yet there are still only a few studies comparing sex differences, with some studies mixing male and female athletes [35]. These conflicting results may be due to the influence of other moderators, such as strength levels [14, 15] and age [34, 36, 37], if we also consider the suggestions from studies examining PAP responses [36-38]. Furthermore, the influence of hormones on myosin light chain phosphorylation should be taken into account [38, 39]. For this reason, identification of athletes' sex and age is also recommended, apart from the specific sport. Further, the physiological profile associated to the demands of each sport (individual vs. team sports; endurance vs. power sports) can importantly influence both PAPE factors and responses [7, 19]. But this moderator is not always considered, specifically in studies that mix athletes from different sports [18, 35].

\section{Applications of PAPE}

\section{Warm-up}

The most widely used application of PAPE strategies is warm-up. In this respect, it is recommended to start all the warm-ups with brief submaximal efforts 
D. Boullosa, Post-activation performance enhancement for practitioners

(e.g. 5 minutes of submaximal running at $50-60 \%$ of maximum heart rate) before performing specific PAPE strategies, to increase body temperature and metabolic responses [8, 25]. Long (i.e. $>15$ minutes) warm-up protocols should be avoided as they can increase central and peripheral fatigue therefore interfering with PAPE responses [40-42]. This is a key aspect to be further explored, given the frequent suggestion of including a number of different exercises (e.g. balance, agility) in the warm-up with the objective of reducing injury risk [43].

PAPE is more often used to enhance performance in explosive or power exercises. Therefore, the athletes that would benefit the most from this strategy would be power athletes [35], such as sprinters [44] or jumpers [45]. But, paradoxically, studies with these populations are scarce. Most PAPE studies have been conducted with recreationally active subjects and team sports athletes $[14,15]$, whose sport performances are not solely dependent on muscle power [46]. This may be problematic for the applicability of most published approaches, reinforcing the suggestion of better characterizing the population of athletes.

Endurance athletes can also benefit from inclusion of PAPE strategies in the warm-up. But, to date, there is still limited evidence with these athletes [26, $32,47]$. Of note, considering the transient duration of PAPE (up to 10 minutes after CAs), it is still to be solved if the pacing improvements during the first splits of an endurance test may affect the total time, which may be related to the duration of the endurance exercise [48-50]. In addition, more studies comparing the effect of different warm-up protocols on both PAPE and cardiometabolic responses are needed, as endurance performance can be also related to oxygen uptake kinetics and perceptual responses [19]. In this respect, it is still to be determined if more specific stimuli for endurance athletes (e.g. prolonged submaximal exercise) [51] would elicit greater PAPE than highintensity brief efforts $[6,32,50]$ or a combination of both stimuli [41]. Importantly, this may be related to the physiological profile of the athletes (e.g. muscle fibre type) [19].

One less well understood application of PAPE refers to the augmented work capacity during the subsequent exercises as a consequence of the augmented force capacity. Two recent studies have shown that high-intensity exercises in the warm-up augmented the total volume of a training session in the bench press [52] (i.e. $3 \times 90 \%$ of the $1 \mathrm{RM}$ ) and the back squat [53] (i.e. $2 \times 90 \%$ of the $1 \mathrm{RM}$ ) exercises. This reinforces the necessity of PAPE protocols, to improve not only acute power performances, but also the total volume of the training session probably as a better potentiation/fatigue balance during the training session.

While the use of PAPE is not very problematic in training settings, the application in competitive settings is more difficult because of limited use of equipment and timing constraints [31, 54]. For this reason, it appears that ballistic exercises [13, 31] and other strategies based on easy-to-use equipment, such as elastic bands [26] or cuffs [27, 31], may be attractive alternatives in competitive settings. For instance, a drop jump exercise can be easily implemented when dropping from a stair or a chair on the floor, while an elastic band with a known load can be fixed to any bar or structure to perform several series of half-squat exercises. However, this is not an easy endeavour, considering the need for individualized approaches while respecting the competitive rules. In addition, other ergogenic approaches, such as the use of passive heat maintenance jackets, should be considered for maintenance of elevated body temperature within an optimal range for performance [9, 31].

\section{Testing and monitoring}

The application of standardized PAPE protocols, with perfectly defined factors and moderators, is mandatory for appropriate comparisons over time (i.e. replicability). Paradoxically, there are no studies in literature clearly showing the evolution of PAPE responses over a competitive season in well-trained athletes. The rationale for this stands on the recognized mediation role of training background and experience on PAPE responses in both power [14] and endurance [55] athletes. To the best of our knowledge, there is only one study [56] showing differences in vertical jump performances after a 40-minute running at the anaerobic threshold, before and after a 3-week specific training period in well-trained runners. The authors suggested a change in the muscle fibre recruitment pattern after the training period; however, these results should be taken with caution as all the dependent variables were estimated from jump performance and not directly measured with electromyography [56].

The identification of an increased PAPE after a training period would be an efficient strategy for detecting changes in fitness over the season and thus positive adaptations, as a greater PAPE would be concurrently related to enhancement of muscle potentiation and lowering of fatigue in response to the same stimuli $[40,57]$. These positive physiological adaptations could be systemic and not only of neuromuscular 
origin, if we consider the recent findings by Guerra et al. [58] of a relationship between overall physical fitness and PAPE responses in professional soccer players.

While this proposal is appealing, performing randomized controlled trials is required for appropriately testing this hypothesis in different sports. In this regard, it might be suggested to use competitive exercises to better identify changes in fitness over the season, or simply to monitor changes in neuromuscular performances and other physiological measures during the same training exercises.

\section{Priming and re-warm-up}

One novel and promising area of intervention and research refers to delayed potentiation responses (i.e. priming and re-warm strategies). Although these strategies would be interesting to any sport, their applicability is more in evidence in team sports because of their competitive rules and schedules.

Priming (i.e. pre-warm-up) strategies performed several hours (up to 48 hours) before can improve highintensity and explosive performances [31]. One recent systematic review and meta-analysis [21] revealed that heavy resistance training and running-based sprinting exercises performed 4-6 hours before competition were the best exercises to improve neuromuscular performances in the context of team sports demanding different locomotor activities. For instance, a brief morning warm-up including some maximum short accelerations may potentiate repeated accelerations during the game in the evening. The greater readiness of athletes with the use of these strategies would potentially be related to hormonal status but this point requires further clarification [21]. In this regard, it seems appealing to propose the existence of a link between PAPE and priming mechanisms; this requires further research as there are a limited number of studies on this topic. As specific movement patterns performed under load appear to be more effective [21], it would seem that other mechanisms than PAP may be involved.

Re-warm-up refers to strategies designed to maintain or recover the elevated body temperature and neuromuscular capacity of a previous warm-up. While body temperature could be passively maintained with heated garments [59], the use of PAPE strategies would seem appropriate to increase muscle performances $[20,59]$. This is especially important during halves of competitive matches in team sports [20].

The implementation of these strategies should be performed with caution, given the complex nature of the competitive demands [46] and the need for different recovery strategies [59]. To the best of our knowledge, there is no evidence exploring the potential summative effect of both priming and re-warm-up strategies based on PAPE. This would be very helpful for optimizing the implementation of these approaches throughout the day of competition and during tournaments.

\section{Complex training}

The use of heavy loads before lighter loads of biomechanical similarity is known as complex training [60]. Recent reviews and meta-analyses have confirmed the long-term effectiveness of this approach for the enhancement of specific sport tasks, such as jumping and sprinting [60-62]. However, there are a number of issues to be considered before implementation. For instance, while this training approach is theoretically based on an expected PAPE effect of the heavy loads on the lighter loads of biomechanical similarity, it appears that these 2 assumptions are false since (1) there are no studies systematically reporting the PAPE effects during each session of the examined interventions; (2) there is no evidence on the superiority of biomechanically similar exercises for PAPE [7]. Further, the use of complex training approaches does not seem to be better than the use of contrast training, which consists of performing first all the heavy loads and subsequently the lighter loads at the end of the training session [60]. This point is important as it suggests that the effectiveness of these approaches may be more based on the combination of different stimuli, independently of the order, than on the use of a single stimulus for training adaptations [61]. From a practical point of view, when considering the need of a sufficient recovery time for the purported PAPE [60] and the necessity of individualized interventions [63], the use of complex training would not seem time-efficient and would be very difficult to be implemented in sports settings. Therefore, until future studies confirm the necessity of PAPE responses during complex training interventions for achieving greater improvements in the long term, there is not sufficient evidence to prefer complex training over contrast training or the use of different training stimuli over different days.

\section{Translating research findings to the 'real world'}

Generalization about potentiation responses is not possible if we consider the complex interactions among the PAPE factors and their moderators. Individualization is therefore mandatory when translating research 
results to the 'real world' on the track, on the field, or in the gym. That is, after identifying the specific literature to fulfil our needs, it is important to check with the athlete if the individual responses agree with expectations based on the literature. For this reason, the new taxonomy [7] can be very helpful to identify the $\mathrm{CA}$, the verification test, and the specific population of athletes, which are the main factors modulating PAPE. However, as the new taxonomy has not been used in most previous literature because it has only recently been published [7], we would need to carefully check the correct identification of the PAPE factors in the articles used for our purposes. This is not always possible, given the poor characterization of athletes in some cases.

As an example, if we are training a group of welltrained male middle-distance runners, we may be interested in a simple and effective exercise to rapidly induce PAPE before an 800-m competition. Thus, any PAPE protocol without the need of a complex setting and with a rapid effect would be preferred over other PAPE protocols. After checking the literature, we have found a few articles suggesting the effectiveness of different ballistic exercises on the running economy and performance of endurance athletes [32, 64, 65]. From these studies, it would seem that the best option would be the use of a set of 5 drop jumps with the best reactive strength index 6 minutes before the competition, as this option is the only one that improved 1000-m performance and pacing (i.e. first 250-m split) in a similar population of elite male runners. This would read as 'post-drop jumps, 1000-m running potentiation, in elite male endurance runners' following the new taxonomy [7]. Thus, considering that the PAPE factors are quite similar between our objectives and those reported in this specific study, we would need then to check if slight differences in the PAPE factors (e.g. only 3 drop jumps) and their moderators (e.g. only 3 minutes before competition) would provide a better performance outcome.

Another example could be with male soccer players requiring an optimal preparedness to perform a change of direction $(\mathrm{CoD})$ test during a training session. After reviewing the existing literature with this population, we identified only 4 studies with different approaches to the problem [66-69]. As these studies use very different CAs (flywheel exercises vs. weighted vest vs. halfsquats) to increase performance in very different $\mathrm{CoD}$ tests, we decided to apply the study with weighted vests [69] as we have the vests in the team and because the increases in $\mathrm{CoD}$ performances are the quickest and greatest reported between studies [66-69]. This PAPE protocol would read as 'post-loaded running, $\mathrm{CoD}$ po- tentiation in male soccer players' following the new taxonomy [7].

An interesting option when checking the literature could be the mix of different approaches for looking for a greater PAPE effect. For instance, combining different resistance and jumping exercises (i.e. French contrast method) resulted in greater jump potentiation than a single CA in a recent study [70]. However, attention should be taken to the summatory effect of both potentiation and fatigue when following this approach.

Finally, to effectively confirm the validity and consistency of the approach selected for our purposes, we could use different mathematical approaches [71] to measure the signal-to-noise ratio after determining the reliability and the error of our specific measures, thus identifying the real PAPE effect.

\section{Conclusions}

In this review, we have briefly presented the current evidence on PAPE factors (i.e. CAs, verification tests, and population of athletes) and their potential moderators (i.e. exercise type and loading, timing; recovery interval, target exercise, performance parameter; training background, age, and sex), as well as other delayed potentiation strategies, such as priming and re-warmup exercises, for their use during training, testing, and competition. The consideration of the PAPE factors, following the new taxonomy, would be very helpful for better identifying the best protocols in the literature for our specific purposes in the field, on an individual basis.

\section{Acknowledgements}

I would like to thank Carl Foster for his edits on the manuscript, and the reviewers who provided insightful comments to improve the quality of this work.

\section{Ethical approval}

The conducted research is not related to either human or animal use.

\section{Disclosure statement}

The author does not have any financial interest and did not receive any financial benefit from this research.

\section{Conflict of interest}

The author states no conflict of interest.

\section{References}

1. Tillin NA, Bishop D. Factors modulating post-activation potentiation and its effect on performance of subse- 
quent explosiveactivities. Sports Med.2009;39(2):147166; doi: 10.2165/00007256-200939020-00004.

2. Zimmermann HB, MacIntosh BR, Dal Pupo J. Does postactivation potentiation (PAP) increase voluntary performance? Appl Physiol Nutr Metab. 2020;45(4): 349-356; doi: 10.1139/apnm-2019-0406.

3. Cuenca-Fernández F, Smith IC, Jordan MJ, MacIntosh BR, López-Contreras G, Arellano R, et al. Nonlocalized postactivation performance enhancement (PAPE) effects in trained athletes: a pilot study. Appl Physiol Nutr Metab. 2017;42(10):1122-1125; doi: 10.1139/ apnm-2017-0217.

4. Blazevich AJ, Babault N. Post-activation potentiation versus post-activation performance enhancement in humans: historical perspective, underlying mechanisms, and current issues. Front Physiol. 2019;10:1359; doi: 10.3389/fphys.2019.01359.

5. Prieske O, Behrens M, Chaabene H, Granacher U, Maffiuletti NA. Time to differentiate postactivation "potentiation" from "performance enhancement" in the strength and conditioning community. Sports Med. 2020;50:1559-1565; doi: 10.1007/s40279-020-01300-0.

6. De Poli RAB, Boullosa DA, Malta ES, Behm D, Lopes VHF, Barbieri FA, et al. Cycling performance enhancement after drop jumps may be attributed to postactivation potentiation and increased anaerobic capacity. J Strength Cond Res. 2020;34(9):2465-2475; doi: 10.1519/JSC.0000000000003399.

7. Boullosa D, Beato M, Dello Iacono A, Cuenca-Fernández F, Doma K, Schumann M, et al. A new taxonomy for postactivation potentiation in sport. Int J Sports Physiol Perform. 2020;15(8):1197-1200; doi: 10.1123/ijspp. 2020-0350.

8. McGowan CJ, Pyne DB, Thompson KG, Rattray B. Warm-up strategies for sport and exercise: mechanisms and applications. Sports Med. 2015;45(11):1523-1546; doi: 10.1007/s40279-015-0376-x.

9. Racinais S, Cocking S, Périard JD. Sports and environmental temperature: from warming-up to heating-up. Temperature. 2017;4(3):227-257; doi: 10.1080/23328 940.2017.1356427.

10. Vandenboom R. Modulation of skeletal muscle contraction by myosin phosphorylation. Compr Physiol. 2017;7(1):171-212; doi: 10.1002/cphy.c150044.

11. Wallace BJ, Shapiro R, Wallace KL, Abel MG, Symons TB. Muscular and neural contributions to postactivation potentiation. J Strength Cond Res. 2019;33(3): 615-625; doi: 10.1519/JSC.0000000000003011.

12. Beato M, McErlain-Naylor SA, Halperin I, Dello Iacono A. Current evidence and practical applications of flywheel eccentric overload exercises as postactivation potentiation protocols: a brief review. Int J Sports Physiol Perform. 2020;15(2):154-161; doi: 10.1123/ ijspp.2019-0476.

13. Maloney SJ, Turner AN, Fletcher IM. Ballistic exercise as a pre-activation stimulus: a review of the literature and practical applications. Sports Med. 2014;44(10): 1347-1359; doi: 10.1007/s40279-014-0214-6.

14. Wilson JM, Duncan NM, Marin PJ, Brown LE, Loenneke JP, Wilson SMC, et al. Meta-analysis of postactivation potentiation and power: effects of conditioning activity, volume, gender, rest periods, and training status. J Strength Cond Res. 2013;27(3):854-859; doi: 10.1519/JSC.0b013e31825c2bdb.

15. Seitz LB, Haff GG. Factors modulating post-activation potentiation of jump, sprint, throw, and upper-body ballistic performances: a systematic review with metaanalysis. Sports Med. 2016;46(2):231-240; doi: 10.1007/ s40279-015-0415-7.

16. Chaouachi A, Poulos N, Abed F, Turki O, Brughelli M, Chamari K, et al. Volume, intensity, and timing of muscle power potentiation are variable. Appl Physiol Nutr Metab. 2011;36(5):736-747; doi: 10.1139/h11-079.

17. Boullosa DA, Abreu L, Beltrame LGN, Behm DG. The acute effect of different half squat set configurations on jump potentiation. J Strength Cond Res. 2013;27(8): 2059-2066; doi: 10.1519/JSC.0b013e31827ddf15.

18. Kobal R, Pereira LA, Kitamura K, Paulo AC, Ramos HA, Carmo EC, et al. Post-activation potentiation: is there an optimal training volume and intensity to induce improvements in vertical jump ability in highly-trained subjects? J Hum Kinet. 2019;66:195-203; doi: 10.2478/ hukin-2018-0071.

19. Boullosa D, Del Rosso S, Behm DG, Foster C. Post-activation potentiation (PAP) in endurance sports: a review. Eur J Sport Sci. 2018;18(5):595-610; doi: 10.1080/ 17461391.2018.1438519.

20. Russell M, West DJ, Harper LD, Cook CJ, Kilduff LP. Half-time strategies to enhance second-half performance in team-sports players: a review and recommendations. Sports Med. 2015;45(3):353-364; doi: 10.1007/ s40279-014-0297-0.

21. Mason B, McKune A, Pumpa K, Ball N. The use of acute exercise interventions as game day priming strategies to improve physical performance and athlete readiness in team-sport athletes: a systematic review. Sports Med. 2020;50(11):1943-1962; doi: 10.1007/s40279-02001329-1.

22. Krzysztofik M, Wilk M, Golas A, Lockie RG, Maszczyk A, Zajac A. Does eccentric-only and concentric-only activation increase power output? Med Sci Sports Exerc. 2020;52(2):484-489; doi: 10.1249/MSS.0000000000 002131.

23. Wilk M, Golas A, Krzysztofik M, Nawrocka M, Zajac A. The effects of eccentric cadence on power and velocity of the bar during the concentric phase of the bench press movement. J Sports Sci Med. 2019;18(2):191-197.

24. Beato M, de Keijzer KL, Fleming A, Coates A, La Spina O, Coratella G, et al. Post flywheel squat vs. flywheel deadlift potentiation of lower limb isokinetic peak torques in male athletes. Sports Biomech. 2020;1-14; doi: 10.1080/14763141.2020.1810750. 
D. Boullosa, Post-activation performance enhancement for practitioners

25. Mina MA, Blazevich AJ, Tsatalas T, Giakas G, Seitz LB, Kay AD. Variable, but not free-weight, resistance back squat exercise potentiates jump performance following a comprehensive task-specific warm-up. Scand J Med Sci Sports. 2019;29(3):380-392; doi: 10.1111/ sms.13341.

26. Low JL, Ahmadi H, Kelly LP, Willardson J, Boullosa D, Behm DG. Prior band-resisted squat jumps improves running and neuromuscular performance in middledistance runners. J Sports Sci Med. 2019;18(2):301-315.

27. Doma K, Leicht AS, Boullosa D, Woods CT. Lunge exercises with blood-flow restriction induces post-activation potentiation and improves vertical jump performance. Eur J Appl Physiol. 2020;120(3):687-695; doi: 10.1007/s00421-020-04308-6.

28. Skurvydas A, Jurgelaitiene G, Kamandulis S, Mickeviciene D, Brazaitis M, Valanciene D, et al. What are the best isometric exercises of muscle potentiation? Eur J Appl Physiol. 2019;119(4):1029-1039; doi: 10.1007/ s00421-019-04092-y.

29. Dello Iacono A, Beato M, Halperin I. The effects of cluster-set and traditional-set postactivation potentiation protocols on vertical jump performance. Int J Sports Physiol Perform. 2020;15(4):464-469; doi: 10.1123/ ijspp.2019-0186.

30. Saez Saez de Villarreal E, González-Badillo JJ, Izquierdo M. Optimal warm-up stimuli of muscle activation to enhance short and long-term acute jumping performance. Eur J Appl Physiol. 2007;100(4):393-401; doi: 10.1007/s00421-007-0440-9.

31. Kilduff LP, Finn CV, Baker JS, Cook CJ, West DJ. Preconditioning strategies to enhance physical performance on the day of competition. Int J Sports Physiol Perform. 2013;8(6):677-681; doi: 10.1123/ijspp.8.6.677.

32. Boullosa D, Abad CCC, Reis VP, Fernandes V, Castilho C, Candido L, et al. Effects of drop jumps on 1000$\mathrm{m}$ performance time and pacing in elite male and female endurance runners. Int J Sports Physiol Perform. 2020;15(7):1043-1046; doi:10.1123/ijspp.2019-0585.

33. Sanchez-Sanchez J, Rodriguez A, Petisco C, RamirezCampillo R, Martínez C, Nakamura FY. Effects of different post-activation potentiation warm-ups on repeated sprint ability in soccer players from different competitive levels. J Hum Kinet. 2018;61:189-197; doi: 10.1515/hukin-2017-0131.

34. Arabatzi F, Patikas D, Zafeiridis A, Giavroudis K, Kannas T, Gourgoulis V, et al. The post-activation potentiation effect on squat jump performance: age and sex effect. Pediatr Exerc Sci. 2014;26(2):187-194; doi: 10.1123/pes.2013-0052.

35. Evetovich TK, Conley DS, McCawley PF. Postactivation potentiation enhances upper- and lower-body athletic performance in collegiate male and female athletes. J Strength Cond Res. 2015;29(2):336-342; doi: 10.1519/ JSC.0000000000000728.

36. Kuu S, Gapeyeva H, Ereline J, Pääsuke M. Twitch contractile properties of plantarflexor muscles in young and middle-aged recreationally physically active and non-active women. Aging Clin Exp Res. 2007;19(1):4854; doi: 10.1007/BF03325210.

37. Belanger AY, McComas AJ, Elder GBC. Physiological properties of two antagonistic human muscle groups. Eur J Appl Physiol Occup Physiol. 1983;51(3):381-393; doi: 10.1007/BF00429075.

38. Simpson CL, Flatman MM, Kim BDH, Bouwmeester NM, Jakobi JM. Increase in post activation potentiation in females following a cycling warmup. Hum Mov Sci. 2018;57:171-177; doi: 10.1016/j.humov.2017.12.003.

39. Lai S, Collins BC, Colson BA, Kararigas G, Lowe DA. Estradiol modulates myosin regulatory light chain phosphorylation and contractility in skeletal muscle of female mice. Am J Physiol Endocrinol Metab. 2016;310(9): E724-E733; doi: 10.1152/ajpendo.00439.2015.

40. Rassier DE, MacIntosh BR. Coexistence of potentiation and fatigue in skeletal muscle. Braz J Med Biol Res. 2000;33(5):499-508; doi: 10.1590/s0100-879x2000 000500003.

41. Tomaras EK, MacIntosh BR. Less is more: standard warm-up causes fatigue and less warm-up permits greater cycling power output. J Appl Physiol. 2011; 111(1):228-235; doi: 10.1152/japplphysiol.00253.2011.

42. Yanci J, Iturri J, Castillo D, Pardeiro M, Nakamura FY. Influence of warm-up duration on perceived exertion and subsequent physical performance of soccer players. Biol Sport. 2019;36(2):125-131; doi: 10.5114/biolsport.2019.81114.

43. Al Attar WSA, Alshehri MA. A meta-analysis of metaanalyses of the effectiveness of FIFA injury prevention programs in soccer. Scand J Med Sci Sports. 2019; 29(12):1846-1855; doi: 10.1111/sms.13535.

44. Linder EE, Prins JH, Murata NM, Derenne C, Morgan CF, Solomon JR. Effects of preload 4 repetition maximum on 100-m sprint times in collegiate women. J Strength Cond Res. 2010;24(5):1184-1190; doi: 10.1519/JSC.0b013e3181d75806.

45. Gołaś A, Wilk M, Stastny P, Maszczyk A, Pajerska K, Zając A. Optimizing half squat postactivation potential load in squat jump training for eliciting relative maximal power in ski jumpers. J Strength Cond Res. 2017;31(11):3010-3017; doi: 10.1519/JSC.000000000 0001917.

46. Boullosa D, Casado A, Claudino JG, Jiménez-Reyes P, Ravé G, Castaño-Zambudio A, et al. Do you play or do you train? Insights from individual sports for training load and injury risk management in team sports based on individualization. Front Physiol. 2020;11:995; doi: 10.3389/fphys.2020.00995.

47. Harat I, Clark NW, Boffey D, Herring CH, Goldstein ER, Redd MJ, et al. Dynamic post-activation potentiation protocol improves rowing performance in experienced female rowers. J Sports Sci. 2020;38(14):1615-1623; doi: 10.1080/02640414.2020.1754110.

48. Barranco-Gil D, Alejo LB, Valenzuela PL, Gil-Cabrera J, Montalvo-Pérez A, Talavera E, et al. Warming up 
before a 20-minute endurance effort: is it really worth it? Int J Sports Physiol Perform. 2020;15(7):964-970; doi: 10.1123/ijspp.2019-0554.

49. Chorley A, Lamb KL. The effects of a cycling warm-up including high-intensity heavy-resistance conditioning contractions on subsequent 4-km time trial performance. J Strength Cond Res. 2019;33(1):57-65; doi: 10.1519/JSC.0000000000001908.

50. Silva RAS, Silva-Júnior FL, Pinheiro FA, Souza PFM, Boullosa DA, Pires FO. Acute prior heavy strength exercise bouts improve the $20-\mathrm{km}$ cycling time trial performance. J Strength Cond Res. 2014;28(9):2513-2520; doi: 10.1519/JSC.0000000000000442.

51. Vuorimaa T, Virlander R, Kurkilahti P, Vasankari T, Häkkinen K. Acute changes in muscle activation and leg extension performance after different running exercises in elite long distance runners. Eur J Appl Physiol. 2006;96(3):282-291; doi: 10.1007/s00421-005-0054-z.

52. Alves RR, Viana RB, Silva MH, Guimarães TC, Vieira $\mathrm{CA}$, Santos D de AT, et al. Postactivation potentiation improves performance in a resistance training session in trained men. J Strength Cond Res. 2019; doi: 10.1519/ JSC.0000000000003367.

53. Conrado de Freitas M, Rossi FE, Colognesi LA, de Oliveira JVNS, Zanchi NE, Lira FS, et al. Postactivation potentiation improves acute resistance exercise performance and muscular force in trained men. J Strength Cond Res. 2018; doi: 10.1519/JSC.000000000000 2897.

54. Docherty D, Hodgson MJ. The application of postactivation potentiation to elite sport. Int J Sports Physiol Perform. 2007;2(4):439-444; doi: 10.1123/ijspp.2.4.439.

55. Boullosa DA, Tuimil JL. Postactivation potentiation in distance runners after two different field running protocols. J Strength Cond Res. 2009;23(5):15601565; doi: 10.1519/JSC.0b013e3181a3ce61.

56. Gervasi M, Calavalle AR, Amatori S, Grassi E, Benelli P, Sestili P, et al. Post-activation potentiation increases recruitment of fast twitch fibers: a potential practical application in runners. J Hum Kinet. 2018;65:69-78; doi: 10.2478/hukin-2018-0021.

57. Boullosa DA, Tuimil JL, Alegre LM, Iglesias E, Lusquinos F. Concurrent fatigue and potentiation in endurance athletes. Int J Sports Physiol Perform. 2011;6(1):82-93; doi: 10.1123/ijspp.6.1.82.

58. Guerra MA Jr, Caldas LC, Souza HL, Tallis J, Duncan MJ, Guimarães-Ferreira L. The effects of physical fitness on postactivation potentiation in professional soccer athletes. J Strength Cond Res. 2020; doi: 10.1519/ JSC.0000000000003711.

59. Silva LM, Neiva HP, Marques MC, Izquierdo M, Marinho DA. Effects of warm-up, post-warm-up, and rewarm-up strategies on explosive efforts in team sports: a systematic review. Sports Med. 2018;48(10):22852299; doi: 10.1007/s40279-018-0958-5.

60. Cormier P, Freitas TT, Rubio-Arias JÁ, Alcaraz PE. Complex and contrast training: does strength and power training sequence affect performance-based adaptations in team sports? A systematic review and metaanalysis. J Strength Cond Res. 2020;34(5):1461-1479; doi: 10.1519/JSC.0000000000003493.

61. Pagaduan J, Pojskic H. A meta-analysis on the effect of complex training on vertical jump performance. J Hum Kinet. 2020;71:255-265; doi: 10.2478/hukin-20190087.

62. Bauer P, Uebellacker F, Mitter B, Aigner AJ, Hasenoehrl T, Ristl R, et al. Combining higher-load and lowerload resistance training exercises: a systematic review and meta-analysis of findings from complex training studies. J Sci Med Sport. 2019;22(7):838-851; doi: 10.1016/j.jsams.2019.01.006.

63. Chen Z-R, Lo S-L, Wang M-H, Yu C-F, Peng H-T. Can different complex training improve the individual phenomenon of post-activation potentiation? J Hum Kinet. 2017;56:167-175; doi: 10.1515/hukin-2017-0034.

64. Blagrove RC, Holding KM, Patterson SD, Howatson G, Hayes PR. Efficacy of depth jumps to elicit a post-activation performance enhancement in junior endurance runners. J Sci Med Sport. 2019;22(2):239-244; doi: 10.1016/j.jsams.2018.07.023.

65. Wei CG, Yu L, Duncan B, Renfree A. A plyometric warm-up protocol improves running economy in recreational endurance athletes. Front Physiol. 2020;11:1 97; doi: 10.3389/fphys.2020.00197.

66. Beato M, De Keijzer KL, Leskauskas Z, Allen WJ, Dello Iacono A, McErlain-Naylor SA. Effect of postactivation potentiation after medium vs. high inertia eccentric overload exercise on standing long jump, countermovement jump, and change of direction performance. J Strength Cond Res. 2019; doi: 10.1519/ JSC.0000000000003214.

67. Beato M, Madruga-Parera M, Piqueras-Sanchiz F, Moreno-Pérez V, Romero-Rodriguez D. Acute effect of eccentric overload exercises on change of direction performance and lower-limb muscle contractile function. J Strength Cond Res. 2019; doi: 10.1519/JSC.000000 0000003359.

68. Petisco C, Ramirez-Campillo R, Hernández D, GonzaloSkok O, Nakamura FY, Sanchez-Sanchez J. Post-activation potentiation: effects of different conditioning intensities on measures of physical fitness in male young professional soccer players. Front Psychol. 2019;10: 1167; doi: 10.3389/fpsyg.2019.01167.

69. Turki O, Dhahbi W, Gueid S, Hmaied S, Souaifi M, Khalifa R. Dynamic warm-up with a weighted vest: improvement of repeated change-of-direction performance in young male soccer players. Int J Sports Physiol Perform. 2020;15(2):196-203; doi: 10.1123/ijspp.2018-0800.

70. Hernández-Preciado JA, Baz E, Balsalobre-Fernández C, Marchante D, Santos-Concejero J. Potentiation effects of the French contrast method on vertical jumping ability. J Strength Cond Res. 2018;32(7):1909-1914; doi: 10.1519/JSC.0000000000002437.

71. Hopkins WG. How to interpret changes in an athletic performance test. Sportscience. 2004;8:1-7. 